JINR E2-95-280, June 1995; hep-th/9506206

\title{
Two-dimensional Quantum-Corrected Eternal Black Hole
}

\author{
Sergey N. Solodukhin* \\ Bogoliubov Laboratory of Theoretical Physics, Joint Institute for Nuclear \\ Research, Head Post Office, P.O.Box 79, Moscow, Russia
}

\begin{abstract}
The one-loop quantum corrections to geometry and thermodynamics of black hole are studied for the two-dimensional RST model. We chose boundary conditions corresponding to the eternal black hole being in the thermal equilibrium with the Hawking radiation. The equations of motion are exactly integrated. The one of the solutions obtained is the constant curvature space-time with dilaton being a constant function. Such a solution is absent in the classical theory. On the other hand, we derive the quantum-corrected metric (2.29) written in the Schwarzschild like form which is a deformation of the classical black hole solution [6]. The spacetime singularity occurs to be milder than in classics and the solution admits two asymptotically flat black hole space-times lying at "different sides" of the singularity. The thermodynamics of the classical black hole and its quantum counterpart is formulated. The thermodynamical quantities (energy, temperature, entropy) are calculated and occur to be the same for both the classical and quantum-corrected black holes. So, no quantum corrections to thermodynamics are observed. The possible relevance of the results obtained to the four-dimensional case is discussed.
\end{abstract}

PACS number(s): 04.60.+n, 12.25.+e, 97.60.Lf, 11.10.Gh

* e-mail: solod@thsun1.jinr.dubna.su 


\section{Introduction}

Interest in the quantum corrections in the gravitational theory is two-fold. At first, it is commonly believed that a successful quantization of gravity will provide us with modifications of the theory which are necessary to avoid space-time singularities typically predicted by a classical theory of gravity [1]. These singularities occur in the Universe and inside black holes under rather general assumptions about properties of the matter and manifest themselves in the unlimited increase in the curvature of the space-time. The classical theory is not applicable near a singularity and, in particular, we cannot believe in its predictions concerning the complete global structure of the space-time. Quantum corrections may completely change the gravitational equations and the corresponding geometry on the Planck scale and drastically modify the classical picture [2]. The main problem on this way is the nonrenormalizability of the Einstein gravity since the straightforward exploiting of the standard perturbation methods leads to an inconsistent quantum theory. However, as a first step, we can consider the semiclassical picture when only matter fields are quantized whereas the gravitational degrees of freedom are treated classically. Quantum matter fields, being integrated out in the functional integral, induce a term additional to the Einstein's one in the action. The extremum of the complete effective action gives us a quantum-corrected solution. Unfortunately, in four dimensions the effective action is not known exactly though it can be derived by a nonlocal polynomial with respect to curvatures [3]. The situation is more hopeful in two dimensions where for the conformal matter the effective action is given by the well-known Polyakov-Liouville term. This was the reason why for the last years the two-dimensional models of gravity have intensively been studied [4]. That the theory predicts the existence of two-dimensional black holes was stated in [5]. Then the black hole type solutions were discovered in the so-called "string-inspired" two-dimensional dilaton gravity [6]. It was believed that in two-dimensional toy models one could resolve the old problems of the black hole evaporation [7] by reducing them to solving differential equations of the semiclassical theory. However, the original model [7] occurred to be not exactly integrable, which resulted in

searching and formulating a number of exactly solvable models [8]-[10]. Russo, Susskind 
and Thorlacius (RST) [10 modified the semiclassical action by a local counterterm with which the theory becomes exactly soluble. This RST model found a wide popularity in the context of different aspects of the black hole evaporation [1] and black hole thermodynamics [12]- 14.

The study of two-dimensional models becomes more exciting due to that the fourdimensional Einstein theory in the spherically symmetric case reduces to an effective two-dimensional theory of the dilaton type [15], [16]. This allows one to find the effective action in the spherically symmetric case and the corresponding quantum deformation of the classical (Schwarzschild) configuration [16].

The other point where the quantum corrections may be important is the thermodynamics of black hole. The most intriguing problem is dynamical explanation of the degrees of freedom inside a hole that are counted by the Bekenstein-Hawking formula [17 relating the entropy of a hole with the area of its horizon (for the review of different approaches see ref. [18]). There has been much interest in this problem [19-27] in the recent literature. One of the ideas is that the entropy of a hole is due to quantum matter excitations propagating inside or just outside the horizon. So the whole black hole entropy can be treated as a quantum correction. It has been shown that it is ultraviolet divergent [19] that can be removed by the standard renormalization of the gravitational constant [20]-25]. (For the discussion of this problem see also refs. 26], 27].) Unfortunately, the classical (tree-level) Bekenstein-Hawking entropy does not have dynamical explanation in this approach.

However, in addition to divergent corrections there might be finite corrections to the thermodynamical quantities (mass, entropy, temperature) that are of high interest since they may be essential at the final stages of the black hole evaporation when mass of the black hole becomes comparable with the Planck mass. The corrections, logarithmically dependent on the mass of a hole, have recently been observed in two [12], [22] and four [28] dimensions by means of the perturbative calculations on the fixed classical background.

The aim of this paper is to give, within the $2 \mathrm{D}$ dilaton gravity modified by the oneloop contributions according to RST [10], the complete and detailed investigation of the 
above-noted problems: form of the quantum-corrected geometry of the eternal black hole and calculation of the corresponding thermodynamical quantities in one loop.

One remark is in order. The RST model is exactly solvable but the solution is uniquely defined only up to the boundary conditions that may essentially change the character and physical interpretation of the solution. This is because the nonlocal nature of the Polyakov-Liouville term the effective action contains an ambiguity corresponding to different choices of the quantum state of the system. The choice made in [10] describes the formation of the black hole from vacuum space-time due to the incoming matter. There is no any Hawking radiation for the vacuum flat space-time stage; the radiation energy-momentum tensor is zero, $T_{\mu \nu}^{r a d}=0$, in the asymptotically flat region. Instead, we are interested in the already formed eternal black hole being in the thermal equilibrium with the Hawking radiation. At infinity, we have asymptotically flat space-time filled by radiation with the energy density $T_{0}^{0, r a d}=\frac{\pi}{6} N T^{2}$. Therefore, our choice of boundary conditions is different from that of [10] to ensure this behavior at infinity.

Our paper is organized as follows. In Section 2. we write down the RST equations, explain our choice of the boundary conditions describing the eternal black hole and find exactly the general solution in the Schwarzschild form. The solution obtained is a quantum deformation of the known classical dilaton black hole [6]. The global structure of the found solution is studied in Section 3. In Section 4., we give some general remarks on formulation of the black hole thermodynamics and calculate the thermodynamical quantities (mass, entropy, temperature) for the classical black hole円 and its one-loop counterpart. The comparison with the previous perturbative calculations [12], [22] is given. The possible relevance to the four-dimensional black hole physics is discussed in Section 5. The results obtained are summarized in the Conclusion.

\section{Eternal black hole solution of the RST model}

\section{A. Action and field equations}

\footnotetext{
${ }^{1}$ The thermodynamics of the classical $2 \mathrm{D}$ dilaton black hole has been previously studied in [29]-[31].
} 
The classical action of the dilaton gravity [6]

$$
I_{0}=\frac{1}{2 \pi} \int_{M} d^{2} x \sqrt{-g} e^{-2 \phi}\left[R+4(\nabla \phi)^{2}+4 \lambda^{2}\right]+\frac{1}{\pi} \int_{\partial M} e^{-2 \phi} k d s
$$

on the quantum level, accordingly to [10], gets modified by the following terms:

$$
I_{1}=-\frac{\kappa}{2 \pi} \int_{M} d^{2} x \sqrt{-g} \phi R-\frac{\kappa}{\pi} \int_{\partial M} \phi k d s
$$

and

$$
I_{2}=-\frac{\kappa}{2 \pi} \int_{M} d^{2} x \sqrt{-g}\left(\frac{1}{2}(\nabla \psi)^{2}+\psi R\right)-\frac{\kappa}{\pi} \int_{\partial M} \psi k d s
$$

where we added in (2.1) and (2.2) the boundary terms determined with respect to the second fundamental form $k$ in order to have the well-defined variational problem. If $n^{\mu}$ is an outward vector normal to the boundary $\partial M$, then $k=\nabla_{\mu} n^{\mu}$. The function $\psi$ is the solution of the equation

$$
\square \psi=R
$$

where $\square=\nabla_{\mu} \nabla^{\mu}$.

The action $I_{2}$ is the Polyakov-Liouville term f incorporating both the Hawking radiation of the scalar matter $N$-multiplet $\left(\kappa=\frac{N}{24}\right)$ and its back-reaction on the black hole geometry. The local term $I_{1}$ (2.2) is added [10] to preserve, on the quantum level, some symmetry of the classical action (2.1). We are working in the semiclassical approximation when only the matter fields surrounding the black hole are quantized while the metric of two-dimensional space-time is still classical. Then, the minimum of the effective action

$$
I=I_{0}+I_{1}+I_{2}
$$

under appropriately defined boundary condition gives us the quantum-corrected black hole configuration.

Varying (2.5) with respect to metric we get the equation $\left(T_{\mu \nu}=2 \frac{\delta I}{\delta g^{\mu \nu}}\right)$ :

$$
T_{\mu \nu} \equiv T_{\mu \nu}^{(0)}+T_{\mu \nu}^{(1)}+T_{\mu \nu}^{(2)}=0
$$

where

$$
T_{\mu \nu}^{(0)}=\frac{1}{\pi} e^{-2 \phi}\left(2 \nabla_{\mu} \nabla_{\nu} \phi-2 g_{\mu \nu}\left(\square \phi-(\nabla \phi)^{2}+\lambda^{2}\right)\right)
$$

\footnotetext{
${ }^{2}$ The reasons for writing the Polyakov-Liouville term in the form (2.3) are analyzed in [32].
} 


$$
\begin{gathered}
T_{\mu \nu}^{(1)}=-\frac{\kappa}{\pi}\left(g_{\mu \nu} \square \phi-\nabla_{\mu} \nabla_{\nu} \phi\right) \\
T_{\mu \nu}^{(2)}=-\frac{\kappa}{2 \pi}\left(\partial_{\mu} \psi \partial_{\nu} \psi-2 \nabla_{\mu} \nabla_{\nu} \psi-g_{\mu \nu}\left(-2 R+\frac{1}{2}(\nabla \psi)^{2}\right)\right),
\end{gathered}
$$

Variation of (2.5) with respect to $\phi$ gives the dilaton field equation

$$
2 e^{-2 \phi}\left(R+4 \square \phi-4(\nabla \phi)^{2}+4 \lambda^{2}\right)=-\kappa R
$$

Taking trace of the energy-momentum tensor (2.6) $T_{\mu \nu} g^{\mu \nu}=0$, we get

$$
2 e^{-2 \phi}\left(\square \phi-2(\nabla \phi)^{2}+2 \lambda^{2}\right)=-\kappa(R+\square \phi)
$$

Comparing (2.10) and (2.11) we come to the equation

$$
(R+2 \square \phi)\left(\kappa-2 e^{-2 \phi}\right)=0
$$

Remarkably, we now have only two possibilities.

The solution of the first type is characterized by the constant value of the dilaton

$$
2 \phi=-\ln \frac{\kappa}{2}=\text { const }
$$

and the constant curvature

$$
R=-2 \lambda^{2}
$$

Then equation (2.6) reduces to the theory of the induced 2D gravity with the cosmological term (see [33], [34]). This de Sitter space-time solution is absent in the classical theory described by the action (2.1). Nevertheless, the value of the curvature (2.14) is "classical" since it does not depend on $\kappa$ characterizing the quantum effects ( $\kappa$ is proportional to the Planck constant $\hbar)$. This constant curvature solution was missed out in the previous consideration of the model. Note that this solution lies completely in the quantum mechanical strong coupling region. Therefore, one could assume that it is an artifact of one loop and it is absent in the full quantum theory. However, one can show that the de Sitter space ( $R=$ const, $\phi=$ const) is still the solution of three-loop $\beta$-function equation in the $D=2 \sigma$-model [35] that can be treated as $2 D$ quantum gravity [36], [37].

\section{B. Choice of boundary conditions}


The second possibility following from eq.(2.12) consists in that the dilaton $\phi$ is a nonconstant function on the two-dimensional manifold. Then, we have from (2.12) the key relation

$$
R=-2 \square \phi
$$

allowing one to integrate exactly all the field equations (2.6)-(2.10). Eq.(2.15) means that the function $\psi$ reads

$$
\psi=-2 \phi+w
$$

where $w$ is the solution of the homogeneous equation, $\square w=0$. The nonlocal nature of the action $I_{2}(2.3)$ is reflected in the dependence on such an arbitrary function $w$. The concrete choice of $w$ is provided by appropriate boundary conditions corresponding to the chosen quantum state of the whole system. One natural choice is to put $\partial_{\mu} \psi=0$ in the asymptotically flat region. This means that asymptotically $T_{\mu \nu}^{(2)}=0$ and, hence, no Hawking radiation is present in the flat Minkowskian space-time. This boundary condition is reasonable in the situation when formation of a hole from flat space-time due to the incoming matter is considered [10].

Instead, here we assume the hole with non-zero mass to be already formed and to be in the equilibrium with the environment of the fluctuating quantum fields behaving like thermal gas at infinity. The geometry of such eternal black hole is deformed by the backreaction effects of this environment. Our choice of the boundary conditions is regulated by the following two requirements: 1) There is no singularity of $\psi\left(\right.$ and $\left.T_{\mu \nu}^{(2)}\right)$ at the horizon for the Hawking temperature $T=T_{H}$. 2) In the asymptotically flat region, the backreaction is negligible and we have the semi-classical picture: flat space-time filled by the thermal Hawking radiation with energy density $T_{0}^{0(2)}=T_{0, t h}^{0}=\frac{\pi}{6} N T^{2}$ and temperature $T$.

As has been shown in ref. 22, the solution of the equation $\square \psi=R$ for metric written in the Schwarzschild gauge

$$
d s^{2}=-g(x) d t^{2}+\frac{1}{g(x)} d x^{2}
$$

3 This relation is present in the classical theory described by the action $I_{0}$ (2.1). The one-loop term $I_{1}$ (2.2) is added in order to preserve this relation on the quantum level. 
takes the form

$$
\psi=-\ln g-b \int_{x} \frac{d x}{g(x)}
$$

and the renormalized energy density of the Hawking radiation is as follows:

$$
T_{0}^{0(2)}=\frac{\kappa}{2 \pi}\left(2 g_{x}^{\prime \prime}-\frac{1}{2 g}\left(g_{x}^{\prime}-b^{2}\right)\right)
$$

The choice of the constant $b$ in (2.18) and (2.19) specifies the quantum state of the system. It has been proposed in [22] that for the system being at the temperature $T=(2 \pi \beta)^{-1}$ the natural choice is $b=\frac{2}{\beta}$. Then, both $\psi\left(2.18\right.$ ) and $T_{0}^{0(2)}(2.19)$ occur to be regular at the horizon $\left(g\left(x_{h}\right)=0\right)$ for the Hawking inverse temperature $\beta=\beta_{H} \equiv 2\left(g^{\prime}\left(x_{h}\right)\right)^{-1}$ and, asymptotically, (2.19) gives the energy density of the thermal bath with temperature $T$ : $T_{0}^{0(2)} \rightarrow T_{0, t h}^{0}=\frac{\pi}{6} N T^{2}$. Thus, we have for the system lying in the box with size $L$ under temperature $T=(2 \pi \beta)^{-1}$ :

$$
\psi=-\ln g-\frac{2}{\beta} \int_{x}^{L} \frac{d x}{g(x)}+\ln g(L)+c
$$

where $c$ is constant.

The form (2.20) for the function $\psi$ is general and valid for an arbitrary 2D theory describing eternal black hole. Being interested in the concrete model (2.5), it is instructive to give (2.20) the semiclassical consideration on the classical black hole configuration [6] which is minimum of the action $I_{0}$ (2.1). It takes the form (2.17) with

$$
g(\phi)=1-a e^{2 \phi}, \quad \phi=-\lambda x
$$

Then, we obtain that at the Hawking temperature, $\beta=\beta_{H} \equiv \frac{2}{g^{\prime}\left(x_{h}\right)}$, the function $\psi(2.20)$ considered on this classical background takes the form (2.16) with $w=$ const:

$$
\psi(x)=-2 \phi(x)+2 \phi(L)+c
$$

where $\phi(L)$ is the value on the boundary $x=L$. Equivalently, (2.22) means the condition $\left.\partial_{x} \psi\right|_{x=L}=2 \lambda$.

The contribution to the entropy due to the Hawking radiation is determined by the value of function $\psi$ on the horizon [13], 38]: $S=-2 \kappa \psi\left(x_{h}\right)$. We obtain for (2.22) that 


$$
S=4 \kappa\left(\phi\left(x_{h}\right)-\phi(L)-\frac{c}{2}\right)
$$

Then, inserting (2.21) we see that this quantity

$$
S=\frac{\pi}{3} N L T-2 \kappa \ln a-2 \kappa c
$$

reproduces the entropy of the thermal bath $S_{t h}=-4 \kappa \phi(L)=\frac{\pi}{3} N L T$ in the box with

size $L$ and temperature $T=\frac{\lambda}{2 \pi}$. The second term in the r.h.s. of (2.23), $4 \kappa \phi\left(x_{h}\right)$ can be interpreted as an addition (correction) to the entropy of the black hole itself. Thus, $\psi$ in the form (2.20) with the boundary condition $\psi(x=L)=0$ (this fixes the indefinite constant in $(2.20), c=0$ ) includes automatically the effects of the thermal bath of the asymptotically flat space-time.

We expect that the semiclassical consideration is correct in the asymptotically flat region. In particular, there we have (2.22) for $\psi$. Therefore, according to our second requirement, we take the gauge (2.22) or, equivalently, $w=$ const in (2.16) in the complete one-loop theory. The condition (2.22) will guarantee, by the way, the regularity of $\psi$ on the horizon.

In two dimensions the Hawking temperature and, correspondingly, energy density of the Hawking radiation at infinity are independent of mass of a hole. Therefore, our requirements 1) and 2) concern only the nonzero mass hole. For flat space-time (zero mass) there are no reasons for the radiation and, hence, we need different boundary condition, namely $\partial_{\mu} \psi \rightarrow 0\left(T_{\mu \nu}^{(2)} \rightarrow 0\right)$ (or, equivalently, $w \rightarrow-2 \lambda x$ in $(2.16)$ ).

\section{Exact integrability}

By taking into account (2.10) and (2.16), 2.22), eq.(2.6) is written in the form

$$
\nabla_{\mu} \nabla_{\nu} F(\phi)=\frac{1}{2} g_{\mu \nu} \square F(\phi)
$$

where $F$ is the function of the dilaton

$$
F(\phi) \equiv \phi-\frac{\kappa}{4} e^{2 \phi}
$$

An equation like (2.24) normally appears in different two-dimensional models of gravity. It means that $\xi_{\mu}=\epsilon_{\mu}{ }^{\nu} \partial_{\nu} F$ is the Killing vector $\left(\nabla_{(\mu} \xi_{\nu)}=0\right)$. This fact essentially simplifies 
the integrating of field equations. Indeed, we may use the variable $x=\frac{1}{Q} F(\phi)$ as spacelike coordinate on the $2 \mathrm{D}$ space-time. Then, it follows from (2.24) that metric takes the Schwarzschild-like form (2.17) and it is static (independent of the time variable $t$ ). The concrete form of the metric function $g(x)$ is found from eq.(2.15) which reads

$$
\partial_{x}^{2} g=2 \partial_{x}\left(g \partial_{x} \phi\right)
$$

By integrating (2.26) it is more convenient to find $g$ as a function of the dilaton $\phi$ under assumption that $\phi(x)$ is given by the equation $Q x=F(\phi)$. Then, we have from (2.26) that

$$
2 g=\partial_{\phi} g+\frac{d}{Q} \partial_{\phi} F(\phi)
$$

where $d$ is constant. The solution of (2.27) is easily found:

$$
g(\phi)=1-a e^{2 \phi}+\kappa \phi e^{2 \phi}
$$

where we have put $d=2 Q$ in order to have $g=1$ in the asymptotically flat region $(\phi \rightarrow-\infty) ; a$ is the integrating constant.

Inserting now (2.17) and (2.28) into (2.10), we get $Q=-\lambda$ for the constant. Finally, the general solution of equations (2.6)-(2.10) in the gauge (2.22) is the following

$$
\begin{aligned}
& d s^{2}=-g(x) d t^{2}+\frac{1}{g(x)} d x^{2}, \\
& -\lambda x=F(\phi) \equiv \phi-\frac{\kappa}{4} e^{2 \phi}, \\
& g(\phi)=1-a e^{2 \phi}+\kappa \phi e^{2 \phi} .
\end{aligned}
$$

Using the identity (2.27) it is easy to check that $\psi$ defined as (2.20) (at $\beta=\beta_{H}$ ) for the quantum-corrected background (2.29) indeed takes the form (2.22). Thus, the whole integration procedure is self-consistent.

As we could expect, the general solution (2.29) does not contain the flat space. Our boundary condition (2.22) assumes the presence of the thermal gas with the nonzero energy density in the asymptotical region that necessary curves the space-time. As a result, at infinity the positive thermal energy density, $T_{0}^{0(2)}=\frac{\kappa \lambda^{2}}{\pi}$, is compensated by the negative energy density of the gravitational fieldf, $T_{0}^{0(0)}=-\frac{\kappa \lambda^{2}}{\pi}$.

\footnotetext{
${ }^{4}$ This is the energy density of the gravitational field described by the metric function $g_{0}=1+\kappa \phi e^{2 \phi}$ that is valid in the asymptotical region.
} 
We see that in the limit $\kappa=0(2.29)$ coincides with the classical black hole solution (2.21). However, asymptotically $(\phi \rightarrow-\infty, x \rightarrow+\infty)$, the last ("quantum") term in (2.29) dominates and the solution goes not to the classical one (2.21) but to that of

(2.29) with $a=0, g \rightarrow g_{0}=1+\kappa \phi e^{2 \phi}$. This solution is asymptotically flat and is a quantum deformation (with $\kappa$ being the deformation parameter) of the classical linear dilaton vacuum. It is an natural reference configuration (instead of the flat space) with respect to which the quantities (like energy) measured at infinity are defined.

\section{Global structure of the quantum corrected space- time}

The dilaton field $\phi$ as a function of $x$ is two-valued. The critical point $\phi_{c r}=\frac{1}{2} \ln \frac{2}{\kappa}$ defined as $F^{\prime}\left(\phi_{c r}\right)=0$ separates its two branches. So in the regions $\phi<\phi_{c r}$ or $\phi>\phi_{c r}, \phi(x)$ is one-valued. We call these regions the $(+)$ and $(-)$ ones, respectively. The derivative with respect to the variable $x$ is defined as $\partial_{x}=-\frac{\lambda}{F^{\prime}(\phi)} \partial_{\phi}$. Therefore, the point $\phi=\phi_{c r}$ is the place where the space-time singularity is present. Indeed, the scalar curvature for the metric 2.29$)$

$$
R \equiv-g_{x}^{\prime \prime}=-\frac{4 \lambda^{2} e^{2 \phi}}{\left(\frac{\kappa}{2} e^{2 \phi}-1\right)^{3}}\left(a-\kappa-\kappa \phi+\frac{\kappa^{2}}{4} e^{2 \phi}\right)
$$

takes infinity at $\phi=\phi_{c r}$.

Thus, the singularity of the classical black hole $(\kappa=0)$, located at $\phi=+\infty$ is now shifted to the finite value of the dilaton, $\phi=\phi_{c r}$.

Other important point characterizing $(2.29)$ is that the flat space-time is not a solution to any parameter $a$. This is obviously due to the boundary condition (2.22) and the fact that back-reaction of the Hawking radiation drastically changes the geometry of spacetime.

The structure of space-time described by the metric (2.29) essentially depends on the value of the integrating constant $a$ (in the next section we relate it with the hole mass).

For $a<a_{c r}=\frac{\kappa}{2}\left(1-\ln \frac{\kappa}{2}\right)$ the metric function $g(\phi)$ is everywhere positive. So no horizon is present and (2.29) describes space-time with naked singularity. 
At the critical value of $a=a_{c r}$ we have $g\left(\phi_{c r}\right)=g^{\prime}\left(\phi_{c r}\right)=0$. Nevertheless, $g(x)$ has a simple zero at $x_{c r}=-\frac{1}{\lambda} F\left(\phi_{c r}\right)$ since $g_{x}^{\prime}\left(x_{c r}\right)=2 \lambda$.

For $a>a_{c r}$ the metrical function $g(\phi)$ has two zeros, $\phi_{h}$ and $\tilde{\phi}_{h}$. However, since $\phi_{h}<\phi_{c r}<\tilde{\phi}_{h}$, the second zero, $\tilde{\phi}_{h}$, is "beyond" the singularity.

Hence, for $a \geq a_{c r}$ in the region $\phi<\phi_{c r}$ the horizon appears. For $a=a_{c r}$ it coincides with the singularity. In the classical case the point $a=0$ separates the solutions with and without horizons, the case $a=0$ corresponding to everywhere regular flat space-time (vacuum of the theory). The quantum-corrected space with $a=a_{c r}$ is also the smoothest among other solutions (2.29). Indeed, for $a>a_{c r}$ the metric function $g(x)$ at $x=x_{c r}$ reads

$$
g(x)=-\frac{2}{\kappa}\left(a-a_{c r}\right)+2 \lambda\left(1+\frac{2}{\kappa}\left(a-a_{c r}\right)\right)\left(x-x_{c r}\right)-\frac{4 \sqrt{\lambda}}{\kappa}\left(a-a_{c r}\right)\left(x-x_{c r}\right)^{\frac{1}{2}}
$$

and both $g_{x}^{\prime}$ and $g_{x}^{\prime \prime}$ are singular at $\phi=\phi_{c r}$, the curvature going to infinity as follows:

$$
R \sim-\frac{\lambda^{2}\left(a_{c r}-a\right)}{\kappa\left(\phi_{c r}-\phi\right)^{3}}=-\frac{\lambda^{\frac{1}{2}}\left(a_{c r}-a\right)}{\kappa\left(x_{c r}-x\right)^{\frac{3}{2}}}
$$

For $a=a_{c r}$ the metric

$$
g(x)=2 \lambda\left(x-x_{c r}\right)+\frac{8}{3} \lambda^{\frac{3}{2}}\left(x-x_{c r}\right)^{\frac{3}{2}}
$$

and the first derivative $g_{x}^{\prime}$ are regular at $\phi=\phi_{c r}$, while the curvature (or second derivative $\left.g_{x}^{\prime \prime}\right)$

$$
R \sim-\frac{\lambda^{2}}{\left(\phi-\phi_{c r}\right)}=-\frac{\lambda^{\frac{3}{2}}}{\left(x-x_{c r}\right)^{\frac{1}{2}}}
$$

One can see that the singularity (3.3) is stronger than (3.5). Generally, the metric (2.29) is smoother in comparison with the classical one. In the latter, the singularity is exponential $R=-4 \lambda^{2} a e^{2 \phi}$ while in the quantum-corrected case curvature grows by power law (3.3), (3.5). Moreover, the classical singularity manifests itself already in the singular behavior of the metric function, $g_{c l} \sim-a e^{2 \phi}$, while the quantum-corrected metric function (2.29) is regular at $\phi=\phi_{c r}$ and only derivatives,$g_{x}^{\prime}$ and $g_{x}^{\prime \prime}$ diverge. This circumstance allows us to formally consider the regions $\phi<\phi_{c r}$ and $\phi>\phi_{c r}$ as different sheets of the same spacetime glued at $\phi=\phi_{c r}$. The coordinate $\phi$ naturally parametrizes both sheets while $x$ is appropriate to giving a coordinate only on one of them. Both the sheets are asymptotically 
flat though the curvature reaches zero differently:

$$
\begin{aligned}
& R \sim-4 \kappa \lambda^{2} \phi e^{2 \phi}, \phi \rightarrow-\infty \quad(+)-\text { sheet } \\
& R \sim-\frac{8 \lambda^{2}}{\kappa} e^{-2 \phi}, \phi \rightarrow+\infty \quad(-)-\text { sheet }
\end{aligned}
$$

For $a>a_{c r}$ every sheet contains an event horizon: $\phi_{h}$ on the $(+)$-sheet and $\tilde{\phi}_{h}$ on the $(-)$-sheet. Remarkably, the derivative of the metric $g_{x}^{\prime}$ is the same for both the horizons and is equal to $g_{x}^{\prime}\left(x_{h}\right)=2 \lambda$.

Of course, this picture looks formal since no any observer can penetrate through the singularity at $\phi=\phi_{c r}$ and appear at the second sheet. However, this picture is mainly the result of the back-reaction effects within the one-loop approximation. We assume that next loops taken into account will make the singularity at $\phi=\phi_{c r}$ smoother preserving the general global structure to be the same. So, having taken the full effective action, which is the result of all loops contributions, the singularity could be expected to vanish completely.

One can find some support to this idea in the study of the exact (non-perturbative) two-dimensional space-time [40] that emerges from string theory as an exact background of the string target space. The analysis of its global structure shows [41] the remarkable picture: two copies of the black hole space-time having an event horizon but no singularity are glued together to form a wormhole bridging two asymptotically flat regions. This is

in agreement with our present consideration. It would be interesting to find the form of the corresponding (non-perturbative) gravitational action of, possibly, dilaton type giving the dynamics of the string target space geometry and possessing this kind of solution.

\section{Thermodynamics, mass and entropy formulas}

The quantum-corrected black hole solution (2.29) resulted from the one-loop quantum effects. Generally, one could expect that these effects lead also to modification of all characteristics of the hole (mass, entropy, temperature) that possesses quantum corrections. This could change thermodynamical relations, say, entropy as a function of mass, etc.

In this section, we study this problem for the RST model and for the quantumcorrected black hole solution found. To seek the completeness we begin our analysis 
with general remarks on the formulation of the black hole thermodynamics and brief description of the thermodynamics of the classical hole (2.21) (see [29], [30], [31], [22]).

\section{A. Formulation of the black hole thermodynamics}

Consider the system (gravity plus matter) at arbitrary temperature $T=(2 \pi \beta)^{-1}$. The thermodynamics of the field system usually has the Euclidean formulation making the Wick rotation $t=\imath \tau$ and supposing for all fields to be periodical with respect to imaginary time $\tau$ with the period $2 \pi \beta=T^{-1}$, where $T$ is temperature of the system.

We define the state of the system as any configuration $\left(\phi(x), g_{\mu \nu}(x)\right)$ satisfying some general conditions: a) $\phi(x)$ and $g_{\mu \nu}(x)$ are real fields on the Euclidean manifold $(t=\imath \tau)$ with abelian isometry along the Killing vector $\partial_{\tau}$ with the period $2 \pi \beta$; $b$ ) There exists a subspace (horizon) which is a fixed point of the isometry; $c)$ metric $g_{\mu \nu}(x)$ is asymptotically flat.

The condition a) in two dimensions means that metric can be written in the form

$$
d s^{2}=g(x) d \tau^{2}+g^{-1}(x) d x^{2}
$$

where $0 \leq \tau \leq 2 \pi \beta$.

From condition $b$ ) it follows that the metric function has zero $g\left(x_{h}\right)=0$ at some point $x=x_{h}$. This means that the system includes black hole with the horizon at $x=x_{h}$. According to $c$ ) the metric function goes to $g(x) \rightarrow 1$ if $x \rightarrow \infty$. It should be noted that any other constraint on the state $\left(\phi(x), g_{\mu \nu}(x)\right)$ is not assumed. In particular, values on the horizon $\phi\left(x_{h}\right), g_{x}^{\prime}\left(x_{h}\right)$ are arbitrary. We only assume that the system includes the nonextremal hole, i.e. $g^{\prime}\left(x_{h}\right) \neq 0$. This is essential that if $\beta \neq \beta_{H} \equiv \frac{2}{g^{\prime}\left(x_{h}\right)}$, then (4.1) describes space with conical singularity on the horizon. This singularity manifests itself in the $\delta$-like contribution to the curvature so that the complete quantity reads [38]:

$$
\bar{R}=2\left(\frac{1-\alpha}{\alpha}\right) \delta\left(x-x_{h}\right)+R, \quad \alpha=\frac{\beta}{\beta_{H}}
$$

where $R=-g^{\prime \prime}$ is the regular part of the curvature. Thus, our statistical ensemble contains both the regular and singular Euclidean metrics?.

\footnotetext{
${ }^{5}$ In this point our approach differs from that developed by York with collaborators [39] in which only regular spacetimes of black hole topology are suggested to form a statistical ensemble. Nevertheless, for quantities calculable at the Hawking temperature the both approaches give the same results.
} 
With respect to the action $I[\beta, g, \phi]$ one can define the free energy $F=\frac{1}{2 \pi \beta} I$ which is functional, $F=F\left[\beta, g_{\mu \nu}(x), \phi(x)\right]$, of the fixed inverse temperature $\beta^{-1}$ and of the state $\left(\phi(x), g_{\mu \nu}(x)\right)$.

Applying the thermodynamical formulas

$$
S=\left(\beta \partial_{\beta}-1\right) I[\beta], \quad E=\frac{1}{2 \pi} \partial_{\beta} I[\beta]
$$

we may calculate the energy and entropy for an arbitrary state $\left(\phi(x), g_{\mu \nu}(x)\right)$ at the fixed $\beta$. These quantities for a system being at the fixed temperature change until a system reaches a thermal equilibrium characterized by the extremum of the free energy $F=E-T S$ (or, equivalently, of the action $\left.I\left[\beta, \phi(x), g_{\mu \nu}(x)\right]\right),(\delta F)_{\beta}=0$. In this variational problem, as it follows from the conditions $a$ )-c), only behavior of fields $\phi, g$ at infinity or at the boundary of the box $(x=L)$ is fixed, $\left.\delta \phi\right|_{x=L}=\left.\delta g\right|_{x=L}=0$. Remarkably, such a equilibrium configuration satisfies the 2-nd law of thermodynamics

$$
\delta E=T \delta S
$$

for small variations around the equilibrium state.

This extreme configuration satisfies the field equations obtained from the action $I$, $\delta_{g} I=\delta_{\phi} I=0$, and for all known cases the extremum is reached on the regular manifold, i.e., the corresponding Hawking temperature coincides with the fixed temperature of the system $\beta=\beta_{H}$. Thus, the state of the system evolves until its Hawking temperature becomes equal to the temperature of the system fixed from the beginning.

The entropy (4.3) taken at $\beta=\beta_{H}$ and satisfying (4.4) is the Bekenstein-Hawking entropy which is determined by total response of the free energy $F$ of the system being in the thermal equilibrium on variation of temperature

\section{B. Thermodynamics of classical black hole}

Apply now these prescriptions to the classical black hole described by the action $I_{0}$ (2.1) (after Wick rotation it changes the overall sign) which on an arbitrary metric with conical singularity takes the form

$$
I_{0}[\beta]=-2 e^{-2 \phi_{h}}\left(1-\frac{\beta}{\beta_{H}}\right)-\frac{1}{2 \pi} \int_{M} d^{2} x \sqrt{-g} e^{-2 \phi}\left[R+4(\nabla \phi)^{2}+4 \lambda^{2}\right]-\frac{1}{\pi} \int_{\partial M} e^{-2 \phi} k d s
$$

\footnotetext{
${ }^{6}$ This follows from the condition $(\delta F)_{\beta}=0$ defining the equilibrium state.
} 
It is a functional of the inverse temperature $\beta$. Consider the configuration which minimizes the action functional (4.5) under $\beta$ fixed. This would be an equilibrium configuration for the given temperature. It should be noted that only a large distance behavior of the metric $g(L) \rightarrow 1, L \rightarrow \infty$ is assumed to be fixed in this variational problem. The functions $g(x), g^{\prime}(x), \phi(x)$ and values on the horizon $\phi\left(x_{h}\right), \quad g^{\prime}\left(x_{h}\right)=\frac{2}{\beta_{H}}$ are supposed to be variable. As a result, the variation with respect to $\delta \phi\left(x_{h}\right)$ gives the constraint

$$
\frac{2}{g^{\prime}\left(x_{h}\right)} \equiv \beta_{H}=\beta
$$

It means that the equilibrium configuration is a regular manifold without conical singularities.

The variation of (4.5) with respect to $\delta g^{\prime}\left(x_{h}\right)$ vanishes automatically due to mutual cancellation of variation of $\beta_{H}$ in the first term defined on the horizon $x=x_{h}$ and that of coming from the second term in the r.h.s. of (4.5). Other (volume) variations give the classical dilaton (eq. 2.10 ) with $\kappa=0$ ) and gravitational $T_{\mu \nu}^{(0)}=0$ equations. The solution is given by (2.21).

For the equilibrium state we have the temperature $T=\frac{1}{2 \pi \beta_{H}}=\frac{\lambda}{2 \pi}$ and the BekensteinHawking entropy of the classical hole

$$
S_{B H}=2 e^{-2 \phi_{h}}=2 a
$$

On the constraint $T_{00}^{(0)}=0$ the energy functional (4.3) reduces to the boundary term

$$
\begin{aligned}
& E=\frac{1}{2 \pi \beta} \int_{\partial M} \frac{2}{\pi} e^{-2 \phi} n^{\mu} \partial_{\mu} \phi d s \\
& =\frac{2}{\pi}\left(e^{-2 \phi} g(x) \phi_{x}^{\prime}\right)_{x=L}
\end{aligned}
$$

where $d s=g^{1 / 2} d x$ is measure induced on the boundary. This quantity is divergent in the limit $L \rightarrow \infty$ for the classical solution (2.29) and, in particular, for the flat space $(g=1, \phi=-\lambda x)$. To regularize the quantity (4.8), one must subtract the flat space contribution. To this aim, we add to the action $I_{0}$ the additional boundary term

$$
I_{0} \rightarrow I_{0}-\frac{2}{\pi} \int_{\partial M} e^{-2 \phi}\left(n_{0}^{\mu} \partial_{\mu} \phi\right) d s
$$

\footnotetext{
${ }^{7}$ Translating the two-dimensional physics to the $4 D$ language it is useful to have in mind the analogy between the dilaton field $\phi$ and the radius $r^{2}$ in the $4 D$ spherically symmetric case, $r^{2} \sim e^{-2 \phi}$ (see the Section 5 for details). Then, (4.7) is written as $S \sim r_{h}^{2}$ that is similar to the known four-dimensional law relating entropy of a hole with the area of the horizon $A_{h}=\pi r_{h}^{2}$.
} 
where the normal vector $n_{0}^{\mu}$ is defined with respect to the flat metric $g=1$. Then, expression for the energy (4.8) gets modified:

$$
E=\frac{2}{\pi}\left(e^{-2 \phi} g^{1 / 2}\left(g^{1 / 2}-1\right) \phi^{\prime}(x)\right)_{x=L}
$$

Substituting solution (2.21) into (4.10) we obtain in the limit $L \rightarrow \infty$

$$
E=\frac{a \lambda}{\pi}
$$

This is the well-known result ([29], [30], [31]) for the mass of the dilaton black hole.

\section{Thermodynamics of the quantum-corrected black hole}

The same approach can be applied to the formulation of thermodynamics of the quantum-corrected hole described by the action (2.5). To get the terms $I_{0}$ and $I_{1}$ on the manifold with conical singularity we can again use formula (4.2) for the complete curvature. One must be more careful, however, with the Polyakov-Liouville term $I_{2}$. It is obtained by integrating the conformal anomaly. It should be noted that the anomaly becomes modified due to the contribution from the conical singularity that really modifies the action $I_{2}$. However, comparison with the exact results shows that up to $(1-\alpha)^{2}$ terms this coincides with naive applying of (4.2) to (2.3).

Taking into account that $\psi=-2 \phi+C$, where $C$ is constant, we finally come to the quantum action $I$ for the metric (4.1) for arbitrary $\beta$ :

$$
\begin{aligned}
& I=-\frac{1}{2 \pi} \int_{M}\left(\left(e^{-2 \phi}+\kappa \phi\right) R+2\left(2 e^{-2 \phi}-\kappa\right)(\nabla \phi)^{2}+4 \lambda^{2} e^{-2 \phi}\right) \\
& -\frac{1}{\pi} \int_{\partial M}\left(e^{-2 \phi}+\kappa \phi\right) k-2\left(1-\frac{\beta}{\beta_{H}}\right)\left(e^{-2 \phi_{h}}+\kappa \phi_{h}\right)+2 \kappa C \chi(M),
\end{aligned}
$$

where $\phi_{h}=\phi\left(x_{h}\right)$ is the value on the horizon, $\beta_{H}=\frac{2}{g^{\prime}\left(x_{h}\right)}$ and

$$
\chi(M)=\frac{1}{4 \pi}\left(\int_{M} R+2 \int_{\partial M} k\right)+\left(1-\frac{\beta}{\beta_{H}}\right)
$$

is the Euler number of the manifold $M$ for arbitrary $\beta$ [22], [38]. One can easily see that $\chi(M)=1$.

\footnotetext{
${ }^{8}$ The conformal anomaly is determined by the heat kernel coefficient $a_{2}, \delta_{\sigma} W=\int_{M} \delta \sigma a_{2}(x)$, which in the presence of the conical singularity has a $\delta$-like contribution from the tip of the cone [42].
} 
Fixing $\beta$ and varying all the variables $g, g^{\prime}, \phi$ we again obtain that equation $\delta_{\phi_{h}} I=0$ gives the condition (4.6) while $\delta_{\phi(x)} I=\delta_{g(x)} I=0$ are the field equations (2.6)-(2.10). So the quantum-corrected equilibrium state is again a regular configuration at the Hawking temperature, $\beta=\beta_{H}$. As it can be seen from (2.29), on the horizon we have $g^{\prime}\left(x_{h}\right)=2 \lambda$. Hence, the temperature of the quantum-corrected hole is the same as for the classical hole, $\beta_{H}=\lambda^{-1}, \quad T_{H}=\frac{\lambda}{2 \pi}$.

From (4.12) we obtain the expression for the entropy

$$
S=2\left(e^{-2 \phi_{h}}+\kappa \phi_{h}\right)-2 \kappa C
$$

where $C=-2 \phi(L)+c($ see (2.22) $)$. The first term in the r.h.s. of (4.13) is defined on the horizon and can naturally be interpreted as the Bekenstein-Hawking entropy of the hole itselff:

$$
S_{B H}=2\left(e^{-2 \phi_{h}}+\kappa \phi_{h}\right)
$$

This result up to an additive constant coincides with that of previously obtained in [12][14]. On the other hand, the second term in (4.13) coincides (for $c=0$ ) with the entropy of the thermal bath with temperature $T_{H}=\frac{2 \pi^{-1}}{\lambda}$ filling the space outside the horizon

$$
S_{t h}=-4 \kappa \phi(L)=\frac{\lambda N L}{6}
$$

Thus, the method of the conical singularity being applied to the quantum effective action gives us both the Bekenstein-Hawking entropy of the quantum-corrected hole and the entropy of thermal gas surrounding the hole. This naturally happens when we put the appropriate boundary condition $\psi(L)=0$ for the function $\psi(x)$ playing an important role in the two-dimensional quantum gravitational physics. In principle, we may subtract the entropy of the gas $S_{t h}$ in the complete entropy putting $c=2 \phi(L) \quad(C=0)$ in (2.22). Generally, different constants $c$ correspond to different choices of the reference point for computation of the system's entropy.

Substituting the solution (2.29) into (4.14) we obtain that the entropy of the quantumcorrected hole coincides with the classical one

$$
S_{B H}=2 a
$$

\footnotetext{
${ }^{9}$ Taking the analogy with the $4 D$ spherically symmetric case, this formula means a modification of the entropy-area relation by a logarithmic correction $S \sim A_{h}-\kappa \ln A_{h}$.
} 
Measuring the entropy with respect to that of vacuum defined as solution (2.29) for $a=a_{c r}$ we obtain

$$
S_{B H}=2\left(e^{-2 \phi_{h}}+\kappa \phi_{h}\right)-2\left(e^{-2 \phi_{c r}}+\kappa \phi_{c r}\right)=2\left(a-a_{c r}\right)
$$

that exactly coincides with the expression derived in [12]-[14].

Using the constraint $T_{00}=0$ we obtain (after short calculations) the expression for the energy

$$
\begin{aligned}
& E=\frac{1}{2 \pi \beta} \frac{1}{2 \pi} \int_{\partial M}\left(2 e^{-2 \phi}-\kappa\right) n^{\mu} \partial_{\mu} \phi d s \\
& =\frac{1}{\pi}\left[\left(2 e^{-2 \phi}-\kappa\right) g \phi^{\prime}\right]_{x=L}
\end{aligned}
$$

Considering (4.18) on (2.29) we see that $E$ is divergent in the limit $L \rightarrow \infty$. Subtracting from the action the same boundary term (4.9) as in the classical case we obtain that the energy

$$
E=\frac{1}{\pi}\left[\left(2 e^{-2 \phi}-\kappa\right) g \phi^{\prime}-2 e^{-2 \phi} g^{1 / 2} \phi^{\prime}\right]_{x=L}
$$

is still divergent

$$
\begin{aligned}
& E=\frac{\lambda a}{\pi}-\frac{\kappa \lambda}{\pi} \phi(L)+\frac{\lambda \kappa}{\pi} \\
& =\frac{\lambda a}{\pi}+\frac{\lambda^{2} N L}{24 \pi}+\frac{\lambda \kappa}{\pi}
\end{aligned}
$$

Up to the last, irrelevant, term eq.(4.20) can be interpreted as a sum of mass of the hole itself,

$$
M=\frac{\lambda a}{\pi}
$$

and of the (divergent) energy of the thermal gas surrounding the hole

$$
E_{t h}=\frac{\lambda^{2} N L}{24 \pi}
$$

We may exclude this contribution of thermal gas and obtain the finite energy if instead of (4.9) we subtract in the action the quantum-corrected boundary term:

$$
I \rightarrow I-\frac{1}{\pi} \int_{\partial M}\left(2 e^{-2 \phi}-\kappa \phi\right) n_{0}^{\mu} \partial_{\mu} \phi
$$

where the normal vector $n_{0}^{\mu}$ is defined with respect to the metric $g_{0}$, the solution (2.29) corresponding to $a=0$, which replaces the flat space at large distances in the quantum 
case. Then the energy reads

$$
E=\frac{1}{\pi}\left[\left(2 e^{-2 \phi}-\kappa\right) g^{1 / 2}\left(g^{1 / 2}-g_{0}^{1 / 2}\right) \phi_{x}^{\prime}\right]_{x=L}
$$

where $g_{0}=1+\kappa \phi e^{2 \phi}$. This quantity is finite and equal to (4.21), $E=M$.

We may measure the mass of the hole with respect to vacuum (solution (2.29) for $\left.a=a_{c r}\right)$. Then, in the boundary term (4.23) the normal vector $n_{0}^{\mu}$ must be defined with respect to the vacuum metric. The expression for energy takes the form (4.24) where now $g_{0}=g_{v a c} \equiv 1-a_{c r} e^{2 \phi}+\kappa \phi e^{2 \phi}$. The resulting energy

$$
E=\frac{\lambda}{\pi}\left(a-a_{c r}\right)
$$

differs form (4.21) by a constant and vanishes for the vacuum configuration $\left(a=a_{c r}\right)$.

There is an alternative derivation of the mass advocated in [31]. Let us assume that all field equations except the gravitational one (2.6) are satisfied. The coordinate invariance of the action (2.5) implies that

$$
\nabla_{\mu} T^{\mu \nu}=0
$$

where $T_{\mu \nu}$ is given by (2.6)-(2.9). Let the gravitational field be static and allow a timelike Killing vector $\xi_{\mu}$. Then, one has

$$
\nabla_{\mu}\left(T^{\mu \nu} \xi_{\nu}\right)=0
$$

In two dimensions (4.26) implies that there exists such a scalar function $M$ that

$$
\nabla_{\alpha} M=-\epsilon_{\alpha \mu} T^{\mu \nu} \xi_{\nu}
$$

In our case $\xi_{\mu}=-\frac{1}{\lambda} \epsilon_{\mu}{ }^{\nu} \partial_{\nu} F(\phi)$ and in the gauge (2.16)-(2.22) after simple calculations we get

$$
M=\frac{\lambda}{\pi}\left(\kappa \phi+e^{-2 \phi}\right)-\frac{1}{\pi \lambda} e^{-2 \phi}(\nabla F)^{2}
$$

When the gravitational field equations $T_{\mu \nu}=0$ (2.6) are satisfied, eq.(4.28) implies that $M=$ const. So (4.28) gives the first integral of the gravitational field equations. Indeed, for the configuration (2.29) we obtain the result (4.21), $M=\frac{\lambda a}{\pi}$. It is worth noting that (4.28) allows one to write the mass formula, relating $M$ with values at the horizon:

$$
M=\frac{\lambda}{\pi}\left(\kappa \phi_{h}+e^{-2 \phi_{h}}\right)
$$




\section{Comparison with perturbative calculations}

The most important conclusion from the above consideration is that in the exact oneloop semiclassical theory the thermodynamical characteristics (the Hawking temperature, mass and entropy) of the quantum-corrected black hole do not possess any quantum corrections. So all the characteristics coincide with the classical ones.

In the previous consideration within the perturbative approach with respect to $\kappa$ (or, equivalently, the Planck constant) the logarithmic, $\ln M$, correction to the entropy has been observed [12], [22]. Therefore, it is worth comparing these perturbative results with the present exact calculation.

On the one-loop level the classical expression for the Bekenstein-Hawking entropy (4.7) as a function of dates on the horizon is modified by the term in (4.14) proportional to $\kappa$. This additional term can be treated as quantum correction, $S_{q}=2 \kappa \phi_{h}$. Expanding the value on the horizon with respect to $\kappa, \phi_{h}=\phi_{h}^{c l}+\kappa \phi_{h}^{q}$, we obtain

$$
S_{q}=2 \kappa \phi_{h}^{c l}=-\kappa \ln \frac{\pi M}{\lambda}
$$

This is the result obtained in [12], 22] and interpreted as a quantum correction to the classical entropy (4.7). The correction (4.30) is essentially due to modification of the entropy formula in the one-loop theory. However, in the consistent approach we must also expand the first (" classical") term $10 S_{c l}=2 e^{-2 \phi_{h}}$ in (4.14) with respect to $\kappa$

$$
S_{c l}=2 e^{-2 \phi_{h}^{c l}}\left(1-2 \kappa \phi_{h}^{q}\right)
$$

From (2.29) we obtain that $2 \phi_{h}^{q}=\phi_{h}^{c l} e^{2 \phi_{h}^{c l}}$. Then, (4.31) reads

$$
S_{c l}=2 e^{-2 \phi_{h}^{c l}}-2 \kappa \phi_{h}^{c l}
$$

This correction is due to the deformation of the geometry of the black hole and of the horizon "location", $\phi_{h}$, in the one-loop theory. We see that both the one-loop modifications: of the entropy formula and black hole geometry, are mutually compensated and the complete entropy, which is the sum of (4.30) and (4.31), remains uncorrected $\square$.

\footnotetext{
${ }^{10}$ I thank V.P.Frolov for this important remark.

${ }^{11}$ This, however, does not exclude that entropy as a function of $\phi_{h}$ gets modified. Taking analogy $r^{2} \sim e^{-2 \phi}$, this means that entropy as a function of the horizon area is really modified by quantum corrections.
} 
There are arguments similar to that leading to (4.30) concerning quantum corrections to entropy of the four-dimensional black hole [28]. However, our present consideration shows that one-loop calculations on the fixed classical background must be accompanied by an analysis of the changing of the black hole geometry due to the back-reaction effects. The latter can be important to make the final conclusion about quantum corrections to the black hole thermodynamics.

\section{Relevance to four dimensions}

In four dimensions we face much more difficult problem. At first, we do not have complete knowledge about the one-loop effective action (more exactly, about its finite nonlocal part). Therefore, modifications of the mass and entropy formulas are not exactly known. On the other hand, attempts to find quantum-corrected solutions minimizing the effective action look hopeless. However, many things are simplified when space-time symmetries are present. For example, the structure of the renormalized energy-momentum tensor for the static spherically symmetric background has been studied in more details [43]. This allows one to find a quantum-corrected black hole configuration in some approximation [44]. In this Section we illustrate the consideration of the previous section in a somewhat different approach developed in [16] and allowing the reduction of the 4D problem to the two-dimensional one.

Indeed, the gravitational Einstein-Hilbert action

$$
I_{g r}=\frac{1}{16 \pi G} \int d^{4} x \sqrt{-g} R^{(4)}
$$

being considered on the four-dimensional spherically symmetric metric of general type:

$$
d s^{2}=\sum_{\alpha, \beta=0}^{1} g_{\alpha \beta}^{(2)} d z^{\alpha} d z^{\beta}+r^{2}(z)\left(d \theta^{2}+\sin ^{2} \theta d \varphi^{2}\right),
$$

is reduced to the effective two-dimensional theory

$$
I_{g r}=\frac{1}{4 G} \int d z^{2} \sqrt{-g^{(2)}}\left(r^{2} R^{(2)}+2(\nabla r)^{2}+2 U(r)\right)
$$

of the dilaton gravity type. The $\frac{r^{2}}{G} \equiv e^{-2 \phi}$ plays the role of the dilaton field. The "dilaton" potential $U(r)$ is constant $U(r)=1$ in the classics. Quantizing only the spherically 
symmetric excitations in the original theory (5.1) we come to the quantum theory of dilaton gravity (5.3). The potential $U(r)$ changes its form due to quantum corrections that was found by solving the corresponding renorm-group equation (for details see [16]). The ultraviolet divergences have been shown to be absorbed in the redefinition of the gravitational coupling $G$. Though in [16] we considered the more general case, we restrict ourselves here, just for illustration, by simple case when one neglects the possible anomalous terms in the quantum version of action (5.3). This approximation is good for enough large mass of hole, $M>10 M_{p l}$. The corrected dilaton potential then reads

$$
U(r)=\frac{r}{\left(r^{2}-l_{p l}^{2}\right)^{\frac{1}{2}}}
$$

where $l_{p l}^{2}=8 G_{r e n}$ is distance of the Planck order. Then the quantum corrected metric takes the form

$$
d s^{2}=-g(r) d t^{2}+\frac{1}{g(r)} d r^{2}+r^{2} d \Omega^{2}
$$

where

$$
g(r)=-\frac{2 M}{r}+\frac{\sqrt{r^{2}-l_{p l}^{2}}}{r}
$$

For $r>l_{p l}$ the classical Schwarzschild black hole solution restores.

Remarkably, the space-time described by the metric (5.5), (5.6) is quite similar to the two-dimensional space-time (2.29) considered in the previous Sections. Indeed, (5.5), (5.6) can be written in the form similar to (2.29):

$$
\begin{aligned}
& d s^{2}=-g(r) d t^{2}+g^{-1}(r) d r^{2}+r^{2}(\phi) d \Omega^{2}, \\
& \frac{r}{l_{p l}}=F(\phi) \equiv \cosh \phi \\
& g(\phi)=\operatorname{th\phi }-2 M(\cosh \phi)^{-1}
\end{aligned}
$$

The classical singularity at $r=0(\phi=+\infty)$ is now shifted to the finite distance $r=l_{p l}$ $\left(\phi=\phi_{c r}\right)$. It has been proposed in [16, that the metric (5.5), (5.6) is formally extended behind the singularity $r=l_{p l}$ to the second asymptotically flat sheet $(\phi \rightarrow+\infty)$ and the singularity becomes smoother due to contributions of ghosts and matter fields. The general structure of the full space-time is similar to that we discussed in Section 3. The essential difference from (2.29) is that there is no extra horizon on the second sheet. 
At large $r>>l_{p l}$ the space-time is no more Ricci flat and the curvature falls as follows:

$$
R^{(4)}=-\frac{2}{r^{2}}\left(\frac{l_{p l}}{r}\right)^{4}
$$

The second term in the r.h.s. of (5.6) at large $r$ falls as $1+O\left(\frac{1}{r^{2}}\right)$. Therefore, the mass $M$ of the hole does not possess any corrections. Nevertheless, the horizon defined by $g\left(r_{h}\right)=0$ is now shifted

$$
r_{h}=\sqrt{(2 M)^{2}+l_{p l}^{2}}
$$

compared with the classical one $r_{h, c l}=2 M$. However, the Hawking temperature $T_{H}=$ $\frac{g^{\prime}\left(r_{h}\right)}{4 \pi}$ remains unchanged

$$
T_{H}=\frac{1}{8 \pi M}
$$

The entropy derived from the action (5.3) reads

$$
S=\frac{A_{h}}{4 G}
$$

where $A_{h}=4 \pi r_{h}^{2}$ is the area of the quantum corrected horizon (5.9) and $G$ is the renormalized gravitational coupling. One can see that $S$ (5.11)being expressed via the corrected quantities takes the classical Bekenstein-Hawking form. In terms of the classical horizon area, however, one observes the constant correction to the classical law

$$
S=\frac{A_{h, c l}}{4 G}+\eta
$$

The concrete value of the constant $\eta=8 \pi$ is irrelevant. Expressions (5.10)-(5.12) illustrate the idea that thermodynamical characteristics of a hole $\left(M, S, T_{H}\right.$ etc.) being expressed in terms of the quantum-corrected quantities may take the classical form. As we have seen, this is realized for the black hole in the two-dimensional RST model. In this Section we found also the partial support to this idea for the $4 D$ Schwarzschild black hole. However, it should be noted that the approximation within which one gets (5.5), (5.6) is well working for large enough mass $M>10 M_{p l}$. In principle, the correction terms $\sim\left(\frac{M_{p l}}{M}\right)^{2}$ to the classical laws could be expected. This needs somewhat more accurate investigations. 


\section{Conclusion}

In this paper, we have analyzed the eternal black hole solution of the two-dimensional RST model giving us an example of exactly solvable one-loop effective theory. The quantumcorrected geometry of the black hole possesses remarkable properties. Though the singularity is still present in the general solution, it becomes milder than in the classical case. Moreover, the equations admit two copies of the asymptotically flat black hole space-time defined on "different sides" of the singularity. One of them (which is behind the singularity) does not have a classical analog. We propose that the complete space-time is a gluing of both copies. It should be noted that a similar picture appears in different quantum models [16], [11]. The singularity is probably absent in the complete quantum theory as it happens for the exact (non-perturbative) black hole background of the string theory [41]. But the global structure of the black hole space-time remains the same.

Generally, quantum corrections are expected to change the thermodynamical relations of a black hole. However, our consideration based on the exact solution of the RST model shows that this does not happen there. The mass, entropy and temperature of the quantum-corrected black hole are the same as in the classical case. So, no quantum corrections! In principle, one can argue that this fact is a feature of this particular model but not a general property of the black hole physics. This problem is worth studying on number of examples. On the other hand, it follows from our consideration that the relation of entropy and dates on the horizon (in our case it is $\phi_{h}$ ) is really modified due to quantum correction (see (4.14)). In terms of the $4 \mathrm{D}$ black hole physics this can be interpreted as the Bekenstein-Hawking entropy is really a more complicated function of the area of the (quantum-corrected) horizon, $S_{B H} \sim \frac{A_{h}}{4}-\kappa \ln A_{h}$, than in the classics. The direct derivation of this result in four dimensions needs further investigation.

\section{Acknowledgments}

I am grateful to V.P.Frolov, W.Israel, R.C.Myers, for valuable discussions. This work was supported in part by grant RFL300 of the International Science Foundation and by grant N 94-02-03665-a of RFFI. 


\section{References}

[1] R.Penrose, Phys.Rev.Lett. 14, 57 (1965); S.Hawking, Proc.R.Soc. (London) A300, 182 (1967); S.Hawking and R.Penrose, Proc.R.Soc. (London) A314, 529 (1970).

[2] V.P.Frolov, G.A.Vilkovisky, Phys.Lett. B106, 307 (1981); V.P.Frolov, M.Markov and V.Mukhanov, Phys.Rev. D41, 383 (1990); E.Fahri and A.Guth, Phys.Lett. B183, 149 (1987); A.Linde, Nucl.Phys. B372, 421 (1992); D.Morgan, Phys.Rev. D43, 3144 (1991); R.H.Brandenberger, Nonsingular cosmology and Planck physics, BROWNHET-985.

[3] A.O.Barvinskii, G.A.Vilkoviskii, Nucl.Phys. B333, 471 (1990); 512 (1990); A.Zelnikov, Phys.Lett. B273, 471 (1991).

[4] J.A.Harvey and A.Strominger, "Quantum Aspects of Black Holes", Enrico Fermi Institute Preprint (1992), hep-th/9209055; S.B.Giddings, "Toy Model for Black Hole Evaporation", UCSBTH-92-36, hep-th/9209113; R.B.Mann, "Lower dimensional black holes: inside and out", WATPHYS-TH-95-02; gr-qc/9501038.

[5] R.B.Mann, A.Shiekh and L.Tarasov, Nucl.Phys. B341, 134 (1992).

[6] G.Mandal, A.Sengupta and S.Wadia, Mod.Phys.Lett. A6, 1685 (1991); E.Witten, Phys.Rev. D44, 314 (1991).

[7] C.G.Callan, S.B.Giddings, J.A.Harvey and A.Strominger, Phys.Rev. D45, R1005 (1992); T.Banks, A.Dabholkar, M.R.Douglas and M.O.'Loughlin, Phys.Rev. D45, 3607 (1992).

[8] A.Bilal and C.Callan, Nucl.Phys., 73 B394 (1993).

[9] S.P. de Alwis, Phys.Rev. D46, 5429 (1992); Phys.Lett. B300, 330 (1993); Phys.Lett. B289, 278 (1992).

[10] J.G.Russo, L.Susskind and L.Thorlacius, Phys.Rev. D46, 3444 (1992); Phys.Rev. D47, 533 (1992). 
[11] L.Susskind, L.Thorlacius and J.Uglum, Phys.Rev.D48, 3743 (1993).

[12] T.M.Fiola, J.Preskill, A.Strominger, S.P.Trivedi, Phys.Rev. D50, 3987 (1994).

[13] R.C.Myers, Phys.Rev. D50, 6412 (1994).

[14] J.D.Hayward, Entropy in the RST model, preprint.

[15] M.McGuigan, C.R.Nappi and S.A.Yost, Nucl.Phys. B375, 421 (1992); O.Lechtenfeld and C.Nappi, Phys.Lett. B288, 72 (1992); D.A.Lowe, Phys.Rev. D47, 2446 (1993).

[16] D.I.Kazakov, S.N.Solodukhin, Nucl.Phys. B429, 153 (1994).

[17] J.D.Bekenstein, Lett.Nuov.Cim. 4, 737 (1972); Phys.Rev. D7, 2333 (1973) ; Phys.Rev. D9, 3292 (1974); S.W.Hawking, Comm.Math.Phys. 43, 199 (1975) .

[18] J.D. Bekenstein, "Do we understand black hole entropy?", qr-qc/9409015.

[19] G.'t Hooft, Nucl. Phys. B256, 727 (1985); M. Srednicki, Phys. Rev. Lett. 71, 666 (1993); L. Bombelli, R. Koul, J. Lee, and R. Sorkin, Phys. Rev. D34, 373 (1986); R.B.Mann, L.Tarasov and A.Zelnikov, Class.Quant.Grav. 9, 1487 (1992); V.Frolov, I.Novikov, Phys.Rev. D48, 4545 (1993); C. Callan, F. Wilczek, Phys.Lett. B 333, 55 (1994); D.Kabat, M.Strassler, Phys.Lett. B329, 46 (1994); S.N. Solodukhin, Phys. Rev. D51, 618 (1995); D.V. Fursaev, Black-hole thermodynamics and renormalization, preprint DSF-32/94, hep-th/9408066; S.P. de Alwis, N.Ohta, On the entropy of quantum fields in black hole backgrounds, COLO-HEP-347; hep-th/9412027; G.Cognola, L.Vanzo and S.Zerbini, One loop quantum corrections to the entropy for a fourdimensional eternal black hole, UTF-342; hep-th/9502006.

[20] L. Susskind and J. Uglum, Phys. Rev. D50, 2700 (1994).

[21] T. Jacobson, Black hole entropy and induced gravity, preprint 1994, gr-qc/9404039.

[22] S.N. Solodukhin, Phys.Rev. D51, 609 (1995).

[23] J.-G. Demers, R.Lafrance and R.C.Myers, Black hole entropy without brick walls, McGill/95-06; gr-qc/9503003. 
[24] D.V.Fursaev, S.N.Solodukhin, On one-loop renormalization of black hole entropy, JINR E2-94-462, hep-th/9412020; S.N.Solodukhin, One-loop renormalization of black hole entropy due to non-minimally coupled matter, hep-th/9504022.

[25] F.Larsen, F.Wilczek, Renormalization of black hole entropy and of the gravitational coupling constant, PUPT 1541, hep-th/9506066.

[26] D.Kabat, Black hole entropy and entropy of entanglement, RU-95-06, hepth/9503016.

[27] J.L.F.Barbón, R.Emparan, On quantum black hole entropy and Newton constant renormalization, PUPT-1529; hep-th/9502155.

[28] D.V.Fursaev, Temperature and entropy of a quantum black hole and conformal anomaly, JINR E2-94-484.

[29] C.Nappi, A.Pasquinucci, Mod.Phys.Lett. A17, 3337 (1992).

[30] G.W.Gibbons and M.Perry, Int.J.Mod.Phys. D1, 335 (1992).

[31] V.P.Frolov, Phys.Rev. D46, 5383 (1992).

[32] V.P.Frolov, W.Israel and S.N.Solodukhin, in preparation.

[33] A.M.Polyakov, Mod.Phys.Lett. A2, 893 (1987); V.G.Knizhnik, A.M.Polyakov and A.B.Zamolodchikov, Mod.Phys.Lett. A3, 819 (1988).

[34] A.H.Chamseddine, M.Reuter, Nucl.Phys. B317, 757 (1988).

[35] A.A.Tseytlin, Phys.Lett. B268, 175 (1991).

[36] J.G.Russo, A.A.Tseytlin, Nucl.Phys. B382, 259 (1992).

[37] T.Banks, J.D.Lykken, Nucl.Phys. B331, 173 (1990).

[38] D.V.Fursaev, S.N.Solodukhin, On description of Riemannian geometry in presence of conical singularities, preprint JINR, E2-95-28, to be published in Phys.Rev. D52 (1995). 
[39] J.W.Jork, Jr., Phys.Rev. D33, 2092 (1986); B.F.Whiting, J.W.Jork, Jr., Phys.Rev.Lett. 61, 1336 (1988); H.W.Braden, J.D.Brown, B.F.Whiting and J.W.Jork, Jr., Phys.Rev. D42, 3376 (1990).

[40] R.Dijkgraaf, H.Verlinde and E.Verlinde, Nucl.Phys. B371, 269 (1992).

[41] M.J.Perry, E.Teo, Phys.Rev.Lett. 70, 2669 (1993).

[42] D.V.Fursaev, Phys.Lett. B334, 53 (1994).

[43] D.N.Page, Phys.Rev. D25, 1499 (1982).

[44] C.O.Lousto, N.Sanchez, Phys.Lett. B212, 411 (1988). 